\title{
Application of Generalized Binomial Distribution Model for Option pricing
}

\author{
Bright O. Osu ${ }^{1, *}$, Samson O. Eggege ${ }^{2}$, Emmanuel J. Ekpeyong ${ }^{3}$ \\ ${ }^{1}$ Department of Mathematics, Michael Okpara University of Agriculture, Umudike, Nigeria \\ ${ }^{2}$ Pope John Paul II Model Secondary School Umunagbor Amagborihitte Ezinitte Mbaise \\ ${ }^{3}$ Department of Statistics, Michael Okpara University of Agriculture, Umudike, Nigeria \\ *Corresponding author: megaobrait@hotmail.com
}

\begin{abstract}
In this work, the Generalized Binomial Distribution (GBD) combined with some basic financial concepts is applied to generate a model for determining the prices of a European call and put options. To demonstrate the behavior of the option prices (call and put) with respect to variables, some numerical examples and graphical illustration have been given in a concrete setting to illustrate the application of the obtained result of the study. It was observed that when there is an increase in strike prices, it leads to decrease in calls option price $C_{(0)}$ and increase in puts option price $P_{(0)}$. Decrease in interest rate leads to decrease in calls option price $P_{(0)}$, and increase in puts option price $P_{(0)}$, and decrease in expiration date leads to decrease in calls option price $C_{(0)}$ and decrease in puts option price $P_{(0)}$. It was also found that the problem of option price can be approached using Generalized Binomial Distribution (GBD) associated with finance terms.
\end{abstract}

Keywords: Generalized Binomial Distribution, European call and put option, portfolio, Stock and Dwass identity

Cite This Article: Bright O. Osu, Samson O. Eggege, and Emmanuel J. Ekpeyong, "Application of Generalized Binomial Distribution Model for Option pricing." American Journal of Applied Mathematics and Statistics, vol. 5, no. 2 (2017): 62-71. doi: 10.12691/ajams-5-2-4.

\section{Introduction}

This paper focuses on a particular type of derivative security known as an option .A contract which gives a buyer the right but obligation to buy or sell an underlying asset or instrument at a specified strike price on a specified date is called an option. To determine its value at any given point in time; one would like to know the value at the time the option is created before the future behavior of the underlying assets is known. Determining an option value is commonly called option pricing. It is also known that both Black-Scholes, CRR model and Binomial model can be used to determine an option's value under a certain conditions on their parameters. If the conditions on the parameters of the generalized Binomial distribution are satisfy, then the generalized Binomial distribution can be combined with financial terms to determine the option value .For example: Chandral et al [1] developed a model for the case of multi period Binomial model of the form

$$
\begin{gathered}
C_{(0)}=\frac{1}{R^{2}} \sum_{j=1}^{2} \frac{2 !}{j !(2-j) !}(\hat{P})^{j}(1-P)^{2-j} \\
\times\left(u^{j} d^{2-j} S_{(0)}-K\right)^{+},
\end{gathered}
$$

where

$$
\left(u^{j} d^{2-j} S_{(0)}-K\right)^{+}=\max \left(u^{j} d^{2-j} S_{(0)}-K, 0\right) .
$$

Cheng - few lee et al [2] showed how the Binomial distribution is combined with some basic finance concepts to generate a model for determining the price of stock option to be of the form;

$$
\begin{aligned}
C=\frac{1}{R^{n}} \sum_{k=0}^{n} & \frac{n !}{k !(n-k) !} p^{k}(1-p)^{n-k} \\
& \times \max \left[0, u^{k} d^{n-k} S-K\right] .
\end{aligned}
$$

Cox et al [3] gave a Binomial model for determining the call price of an option of the form.

$$
\begin{aligned}
C= & \sum_{j=0}^{n} \frac{n !}{j !(n-j) !} p^{j}(1-p)^{n-j} \\
& \quad \times \max \left[0, u^{j} d^{n-j} S-K\right] / r^{n},
\end{aligned}
$$

where $r$ is the interest rate, $\mathrm{n}$ is the number of years for the option to expire, $\max \left[0, U^{j} d^{n-j} S-K\right]$ is the payoff value and $j=0,1,2, \ldots n$.

With a little additional effort Cox et al [3] gave a complete formula in more convenient way to be of the form below for all $j \geq a \max \max \left[0, u^{j} d^{n-j} S-K\right]=$ $u^{j} d^{n-j} S-K$, where $a$ stand for the mimimum number of upward moves of the stock. That is

$$
\begin{gathered}
C=\sum_{j=a}^{n} \frac{n !}{j !(n-j) !} p^{j}(1-p)^{n-j} \\
\times\left[u^{j} d^{n-j} S-K\right] / r^{n},
\end{gathered}
$$


where $r$ is the risk free rate $p$ and $1-p$ are the neutral probabilities, $u$ is the rate at which the stock prices go up and $d$ is the rate at which the stock prices go down, $k$ is the strike price and $n$ is a positive integer.

It can be seen clearly in equation (3) that the parameters satisfy the following conditions
I. $p+(1-p)=1$
II. $1-p=q$

III. $0 \leq p \leq 1$.

If the above conditions on the parameters of Binomial model are also satisfied by the generalized Binomial distribution then the generalized Binomial can be combined with financial terms to determine the call price of an option.

In this paper we use a generalized Binomial distribution together with financial terms to evaluate and monitor the behavior of a call and put option with respect to variables in comparison with Cox et al [3].

The proposed model is of the form

$$
\begin{aligned}
& C_{(0)}=\frac{1}{R^{N}} \sum_{x}^{N}\left(\begin{array}{c}
N \\
x
\end{array}\right) \frac{\hat{A}^{x} \hat{B}^{N-x}}{(\hat{A}+\hat{B})^{N}} C_{N}(x) \\
& =\frac{1}{R} \sum_{x}^{N}\left(\begin{array}{c}
N \\
x
\end{array}\right) \frac{\hat{A}^{x} \hat{B}^{N-x}}{(\hat{A}+\hat{B})^{X+N-X}} C_{N}(x) \\
& =\frac{1}{R^{N}} \sum_{x}^{N}\left(\begin{array}{c}
N \\
x
\end{array}\right)\left(\frac{\hat{A}}{\hat{A}+\hat{B}}\right)^{N}\left(1-\frac{\hat{A}}{\hat{A}+\hat{B}}\right)^{N-x} C_{N}(x) .
\end{aligned}
$$

That is

$$
C_{(0)}=\frac{1}{R^{N}} \sum_{x}^{N}\left(\begin{array}{l}
N \\
x
\end{array}\right)\left(\frac{\hat{A}}{\hat{A}+\hat{B}}\right)^{N}\left(1-\frac{\hat{A}}{\hat{A}+\hat{B}}\right)^{N-x} C_{N}(x) .
$$

where

$$
\begin{aligned}
& C_{N}(x) \\
& =\max \left[\begin{array}{l}
u(u-\alpha)(u-2 \alpha) \ldots u-(x-1) \\
\alpha[d(d-\alpha)(d-2 \alpha) \ldots d-(d-n-x-1)] \\
\alpha S_{(0)}-K
\end{array}\right], \\
& x=0,1,2 \ldots N \text { and } \alpha=0
\end{aligned}
$$

It should be noted that when $\alpha=0$, the proposed model reduces to Cox et al [3], where $R$ is the risk free rate, $\frac{\hat{A}}{\hat{A}+\hat{B}}$ and $1-\frac{\hat{A}}{\hat{A}+\hat{B}}$ is the neutral probabilities, $C_{N}(x)$ denote the pay off values and $N$ is a positive integer that denote the number of years to expiration of the option.

It can be seen below that the parameters in equation(5) satisfy the same conditions in equation (3) above.

$$
\begin{aligned}
& \text { i. } \frac{\hat{A}}{\hat{A}+\hat{B}}+1-\frac{\hat{A}}{\hat{A}+\hat{B}}=1 \\
& \text { ii. } 1-\frac{\hat{A}}{\hat{A}+\hat{B}}=\frac{\hat{B}}{\hat{A}+\hat{B}} \\
& \text { iii. } 0 \leq \frac{\hat{A}}{\hat{A}+\hat{B}} \leq 1 .
\end{aligned}
$$

\section{Method}

The tools for giving the result are the generalized Binomial distribution, Dwass identity with financial terms and Wealth Equation.

The Generalized Binomial distribution in this study was first presented by Dwass (1979). It is a discrete distribution that depends on four parameters $\hat{A}, \hat{B}, \mathrm{~N}$ and $\alpha$, where $\mathrm{A}$ and $\mathrm{B}$ are positive, $\mathrm{N}$ is a positive integer and $\alpha$ is an arbitrary real number, satisfying $(N-1) \leq \hat{A}+\hat{B}$. And Tereapabolan [1] gave Dwass identity of the form $\mathrm{x}^{(\mathrm{i})}=\mathrm{x}(\mathrm{x}-\alpha) \ldots .(\mathrm{x}-(\mathrm{i}-1) \alpha)$.

Let $X$ be the generalized Binomial random variable. Then following Terepabolan [1], its probability function is of the form

$$
\begin{aligned}
& P_{X}(x)=\left(\begin{array}{c}
N \\
x
\end{array}\right) \frac{\left\{\begin{array}{l}
{[A(A-\alpha) . s .(A)-(x-1) \alpha]} \\
\times[B(B-\alpha) . .(B-(n-x-1) \alpha)]
\end{array}\right\}}{\left[\begin{array}{l}
(A+B)(A+B-\alpha) \ldots . . \\
\times(A+B-(N-1) \alpha)
\end{array}\right]} \\
& =\left(\begin{array}{l}
N \\
x
\end{array}\right) \frac{A^{(x)} B^{(N-x)}}{(A+B)^{(N)}}, x=0, N .
\end{aligned}
$$

Tereapabolan and Wongkasem [10] pointed out the three special cases of the distribution in (6) by

i. If $\alpha=0$, it reduces to Binomial distribution with parameters $n$ and $\frac{A}{A+B}$

ii. If $\alpha>0$ it reduces to hypergeometric distribution with parameters $A, B, n$ and $\alpha$ and, some integers $\frac{A}{\alpha}$ and $\frac{B}{\alpha}$

iii. If $\alpha<0$ the result of (6) is pòlya distribution with parameters $A, B, n$ and $\alpha$.

In finance the $\frac{A}{A+B}$ and $1-\frac{A}{A+B}$ are regarded as the neutral probabilities denoted by $\frac{\hat{A}}{\hat{A}+\hat{B}}$ and $1-\frac{\hat{A}}{\hat{A}+\hat{B}}$, therefore Generalized Binomial distribution applied to finance is expressed in the form

$$
\begin{aligned}
& P_{X}(x) \\
& =\left(\begin{array}{l}
N \\
x
\end{array}\right) \frac{\left\{\begin{array}{l}
{[\hat{A}(\hat{A}-\alpha) . .(\hat{A})-(x-1) \alpha]} \\
\times[\hat{B}(\hat{B}-\alpha) \ldots .(\hat{B}-(N-x-1) \alpha)]
\end{array}\right\}}{(\hat{A}+\hat{B})(\hat{A}+\hat{B}) \ldots . .(\hat{A}+\hat{B}-(N-1) \alpha)} \\
& =\left(\begin{array}{c}
N \\
x
\end{array}\right) \frac{\hat{A}^{(x)} \hat{B}^{(N-x)}}{(\hat{A}+\hat{B})^{(N)}}, x=0, N .
\end{aligned}
$$

For Wealth Equation Stockbridge [7] introduced a powerful and general equation for replicating portfolios with the following assumptions: 
i. The initial values of the stock is $S_{(0)}\left(S_{(0)}\right.$ is the stock price at $\mathrm{t}=0$ ).

ii. At the end of the period, the prices is either going up or down with factors $u$ and $d$ that is, $u S_{(0)}$ with probability $\frac{\hat{A}}{\hat{A}+\hat{B}}$ or $d S_{(0)}$ with probability $\frac{\hat{B}}{\hat{A}+\hat{B}}$ which satisfies $0<\frac{\hat{A}}{\hat{A}+\hat{B}}<1$, where $u$ and $d$ are the factors of going up and down respectively.

iii. The movement can also be traced from a view point of tossing a die, which results to a head and tail. If it results to a head at a time $t=1$, we have $S_{1}(H)=$ $u S_{(0)}$ and if it results to a tail at a time $t=1$, we have $S_{1}(T)=d S_{(0)}$.

iv. One dollar invested in the money market at time zero will yield $1+r$ dollar at time one, where $r$ is the interest rate. Conversely one dollar borrowed from the money market at time zero will result in a debt of $1+r$ at time one.

v. The price either increases, by $u>1$ or will decrease by $d<1$.

vi. The price of an option is dependent on the following variables:
a. The strike price $\mathrm{K}$
b. The expire time $\mathrm{T}$
c. The risk free rate $r$
d. The underlying price $S_{(0)}$

Lemma 2.1: For $u>1+r>d>0$, a risk neutral probability $\frac{\hat{A}}{\hat{A}+\hat{B}}=\left(\frac{R-u}{u-d}\right)$, and no arbitrage principle exist if the following holds

$$
\begin{aligned}
& \text { a. } \underbrace{}_{\frac{\hat{A}}{\hat{A}+\hat{B}}}\left(S_{(2)}\right)=R^{2} S_{(0)} \text {, where } R \text { denote the interest } \\
& \text { rate }
\end{aligned}
$$$$
\text { b. } \sum_{l=1}^{3} \frac{\hat{A}}{\hat{A}+\hat{B}}=1 \forall l=1,2,3
$$$$
\text { c. } \frac{\hat{A}}{\hat{A}+\hat{B}}>0 \text {. }
$$

Proof: For $S_{(2)}$ implies $t=2$ and

$$
\begin{aligned}
& \left(\frac{\hat{A}}{\hat{A}+\hat{B}}+1-\frac{\hat{A}}{\hat{A}+\hat{B}}\right)^{2} \\
& =\left(\frac{\hat{A}}{\hat{A}+\hat{B}}\right)^{2}+2 \frac{\hat{A}}{\hat{A}+\hat{B}}\left(1-\frac{\hat{A}}{\hat{A}+\hat{B}}\right)+\left(1-\frac{\hat{A}}{\hat{A}+\hat{B}}\right)^{2} .
\end{aligned}
$$

Defining

$$
\frac{\hat{A}}{\hat{A}+\hat{B}_{1}}=\left(\frac{\hat{A}}{\hat{A}+\hat{B}}\right)^{2}, \frac{\hat{A}}{\hat{A}+\hat{B}_{2}}=2 \frac{\hat{A}}{\hat{A}+\hat{B}}\left(1-\frac{\hat{A}}{\hat{A}+\hat{B}}\right)
$$

and

$$
\frac{\hat{A}}{\hat{A}+\hat{B}_{3}}=\left(1-\frac{\hat{A}}{\hat{A}+\hat{B}}\right)^{2} \text {. }
$$

Now

$$
\begin{aligned}
& E_{\frac{\hat{A}}{\hat{A}+\hat{B}_{i}}}\left(S_{(2)}\right) \\
& =\left[\begin{array}{l}
\left(\frac{\hat{A}}{\hat{A}+\hat{B}}\right)^{2} u^{2} S_{(0)}+2 \frac{\hat{A}}{\hat{A}+\hat{B}}\left(1-\frac{\hat{A}}{\hat{A}+\hat{B}}\right) u d S_{(0)} \\
+\left(1-\frac{\hat{A}}{\hat{A}+\hat{B}}\right)^{2} d S_{(0)}
\end{array}\right] \\
& =\left[\begin{array}{l}
\left(\frac{\hat{A}}{\hat{A}+\hat{B}}\right)^{2}+2 \frac{\hat{A}}{\hat{A}+\hat{B}}\left(1-\frac{\hat{A}}{\hat{A}+\hat{B}}\right) u d \\
+\left(1-\frac{\hat{A}}{\hat{A}+\hat{B}}\right)^{2} d
\end{array}\right] S_{(0)} \\
& =S_{(0)}\left[\frac{\hat{A}}{\hat{A}+\hat{B}} u+1-\frac{\hat{A}}{\hat{A}+\hat{B}} d\right]^{2} \\
& =S_{(0)}\left[\frac{R-d}{U-d} U+\left(1-\frac{u-R}{u-d}\right) d\right]^{2} \\
& =S_{(0)}\left[\frac{R u-d u+d u-R d}{U-d}\right]^{2} \\
& =S_{(0)}\left[\frac{R u-R d}{U-d}\right]^{2}=S_{(0)} R^{2}\left[\frac{u-d}{u-d}\right]^{2} \text {. } \\
& E_{\frac{\hat{A}}{\hat{A}+\hat{B}_{i}}}\left(S_{(2)}\right)=S_{(0)} R^{2} .
\end{aligned}
$$

Defining

$$
\frac{\hat{A}}{\hat{A}+\hat{B}_{1}}=\left(\frac{\hat{A}}{\hat{A}+\hat{B}}\right)^{2}, \frac{\hat{A}}{\hat{A}+\hat{B}_{2}}=2 \frac{\hat{A}}{\hat{A}+\hat{B}}\left(1-\frac{\hat{A}}{\hat{A}+\hat{B}}\right)
$$

and $\frac{\hat{A}}{\hat{A}+\hat{B}_{3}}=\left(1-\frac{\hat{A}}{\hat{A}+\hat{B}}\right)^{2}$ we have

$$
\sum_{l=1}^{3}\left(\frac{\hat{A}}{\hat{A}+\hat{B}}\right)^{2}+2 \frac{\hat{A}}{\hat{A}+\hat{B}}\left(1-\frac{\hat{A}}{\hat{A}+\hat{B}}\right)+\left(1-\frac{\hat{A}}{\hat{A}+\hat{B}}\right)^{2} .
$$

And

$$
\sum_{i=1}^{3} \frac{\hat{A}}{\hat{A}+\hat{B}_{i}}=\left[\begin{array}{l}
\left(\frac{\hat{A}}{\hat{A}+\hat{B}}\right)^{2}+2 \frac{\hat{A}}{\hat{A}+\hat{B}}-2\left(\frac{\hat{A}}{\hat{A}+\hat{B}}\right)^{2} \\
+\left(1-\frac{\hat{A}}{\hat{A}+\hat{B}}\right)^{2}
\end{array}\right]
$$

$$
\begin{aligned}
& {\left[\left(\frac{\hat{A}}{\hat{A}+\hat{B}}\right)^{2}+2 \frac{\hat{A}}{\hat{A}+\hat{B}}-2\left(\frac{\hat{A}}{\hat{A}+\hat{B}}\right)^{2}\right]} \\
& +1-2 \frac{\hat{A}}{\hat{A}+\hat{B}}+\left(\frac{\hat{A}}{\hat{A}+\hat{B}}\right)^{2} \\
& =\left[\left(\frac{\hat{A}}{\hat{A}+\hat{B}}\right)^{2}-\left(\frac{\hat{A}}{\hat{A}+\hat{B}}\right)^{2}+1\right]=1
\end{aligned}
$$




$$
\sum_{i=1}^{3} \frac{\hat{A}}{\hat{A}+\hat{B}_{i}}=1
$$

Now it can be clearly seen that $\frac{\hat{A}}{\hat{A}+\hat{B}_{i}}>0$.

Lemma 2.2: If no arbitrage principle hold then

$$
C_{(t)}=V_{\varnothing}(t) \text { for } \forall t \in\{0\} \text {. }
$$

Lemma 2.3: Let $T \in\{1\}$ and $\emptyset=\left(x_{0}, y_{0}\right) \in R^{2}$, such that $V_{\emptyset}(T)=C_{(T)}$; where $x_{0}$ and, $y_{0}$ are the number of shares of the stock and the unit of the bond respectively.

Then the following holds.

$$
\begin{gathered}
V_{\varnothing}(T)=\left\{\begin{array}{l}
x_{0} u s_{(0)}+y_{0}(1+r) B_{(0)} \\
\text { with probability } \frac{\hat{A}}{\hat{A}+\hat{B}} \\
x_{0} d S_{(0)}+y_{0}(1+r) B_{(0)} \\
\text { with the probability } 1-\frac{\hat{A}}{\hat{A}+\hat{B}}
\end{array}\right. \\
C_{T}=\left\{\begin{array}{l}
C_{u} \text { with } \frac{\hat{A}}{\hat{A}+\hat{B}} \\
C_{d} \text { with } 1-\frac{\hat{A}}{\hat{A}+\hat{B}}
\end{array}\right.
\end{gathered}
$$

Lemma 2.3: Let $u>1+r>d>0$, with $\frac{\hat{A}}{\hat{A}+\hat{B}}=\frac{1+r-d}{u-d}$ and $\frac{\hat{B}}{\hat{A}+\hat{B}}=\frac{u-1+r}{u-d}$. Let $C_{t}$ be a derivative security paying off at $t=2$. We deduce the following equation called Wealth Equation

$$
V_{t+1}=a_{t} S_{t+1}+1+r\left(C_{t}-a_{t} S_{t}\right)=C_{t+1}
$$

for $t=T-1, T-2 \ldots ., 1,0$ then $C_{t+1}=V_{t+1}$.

\section{Main Result}

The following theorem present a generalized Binomial distribution model for option pricing in term of $t=$ 0 and $t=T$

Theorem 3.1: For $\Phi=\left(x_{0}, y_{0}\right) \in \mathbb{R}^{2}$, where $x_{0}$ is the number of shares of stock and $y_{0}$ is the unit of the bond at time $t=0$, then there exist value of $x_{0}$ and $y_{0}$ such that the wealth of the portfolio at timet $=0$ and $t=T$, is

$$
V_{\Phi(t)}=C_{(t)} .
$$

And for $t \in\{0\}$ equation (ii) hold and also for $T \in \mathbb{N}$ equation (iii) hold

$$
\begin{aligned}
& V_{\Phi}(t)=x_{0} S_{(0)}+y_{0} B_{(0)}, \\
& V_{\Phi}(T)=x_{0} S_{(0)}+y_{0} B_{(0)} .
\end{aligned}
$$

Here $V_{\Phi}(t)$ denote the replicating portfolio at $\mathrm{t}=0$, and $V_{\Phi}(T)$ denote the replicating portfolio at maturity, for $T=1,2,3 \ldots$.

\section{Proof}

Taking $T=1$ it gives $V_{\Phi}(1)=C_{(1)}$ then

$$
V_{\Phi}(1)=\left\{\begin{array}{l}
x_{0} u S_{(0)}+y_{0}(1+r) B_{(0)}=C_{u} \\
x_{0} d S_{(0)}+y_{0}(1+r) B_{(0)}=C_{d}
\end{array} .\right.
$$

Letting $\quad \frac{A}{A+B}=\frac{\hat{A}}{\hat{A}+\hat{B}}, \quad \frac{B}{A+B}=1-\frac{\hat{A}}{\hat{A}+\hat{B}} \quad$ and $R=r+1$ then

$$
V_{\Phi}(1)=\left\{\begin{array}{l}
x_{0} u S_{(0)}+y_{0} R B_{(0)}=C_{u} \\
x_{0} d S_{(0)}+y_{0} R B_{(0)}=C_{d}
\end{array} .\right.
$$

Solving the equations (13) smultaneously, we obtain

$$
x_{0}=\frac{C_{u}-C_{d}}{(u-d) S_{(0)}} .
$$

And substituting $x_{0}$ into equation (13) which is the number of shares of stock at $T=1$, we further obtain

$$
\begin{aligned}
& \frac{C_{u}-C_{d}}{(U-d) S_{(0)}} u S_{(0)}+y_{0} R B_{(0)}=C_{u} \\
& \Rightarrow y_{0} R B_{(0)}=C_{u}-\frac{C_{u}-C_{d}}{(U-d) S_{(0)}} u S_{(0)} \\
& =\frac{C_{u}}{1}-\frac{C_{u}-C_{d}}{(u-d) S_{(0)}} u S_{(0)}=\frac{u C_{u}-d C_{u}-u C_{u}+u C_{d}}{(u-d)}
\end{aligned}
$$

Which implies that

$$
y_{0}=\frac{u C_{d-d C_{d}}}{R B_{(0)(U-d)}},
$$

which gives the unit of the bond at $T=1$.

From the fact that $V_{\Phi}(t)=x_{0} S_{(0)}+y_{0} B_{(0)} \forall t \in 0$ $=V_{\Phi}(0)=x_{0} S_{(0)}+y_{0} B_{(0)}$, where $S_{(0)}$ and $B_{(0)}$ denote the price of the stock and Bond respectively at $t=0$

$$
\begin{aligned}
& V_{\Phi}(0)=x_{0} S_{(0)}+y_{0} B_{(0)} \\
& =\frac{C_{u}-C_{d} S_{(0)}}{(u-d) S_{(0)}}+\frac{u C_{d}-d C_{u} B_{(0)}}{R(u-d) B_{(0)}},
\end{aligned}
$$

or

$$
\begin{aligned}
& V_{\Phi(0)}=\frac{C_{u}-C_{d}}{(u-d)}+\frac{u C_{d}-d C_{u}}{R(u-d)} \\
& \Rightarrow V_{\Phi}(0)=\frac{1}{R}\left[\frac{R C_{u}-R C_{d}+u C_{d}-d C_{u}}{(u-d)}\right] \\
& \Rightarrow V_{\Phi}(0)=\frac{1}{R}\left[\frac{R-d}{u-d} C_{u}+\frac{u-R}{u-d} C_{d}\right]
\end{aligned}
$$

where $\frac{R-d}{u-d}=\frac{\hat{A}}{\hat{A}+\hat{B}}$ and $\frac{u-R}{u-d}=1-\frac{\hat{A}}{\hat{A}+\hat{B}}$ substituting into equation(16) we have

$$
V_{\Phi}(0)=\frac{I}{R}\left[\frac{\hat{A}}{\hat{A}+\hat{B}} C_{u}+\frac{\hat{B}}{\hat{A}+\hat{B}} C_{d}\right]
$$


Since no arbitrage principle holds, it implies $V_{\Phi}(0)=$ $C_{(0)}$ by lemma 2.1. Thus

$$
C_{(0)}=\frac{I}{R}\left[\frac{\hat{A}}{\hat{A}+\hat{B}} C_{u}+\frac{\hat{B}}{\hat{A}+\hat{B}} C_{d}\right] .
$$

Now we are interested in the case where there is more than one period for the option to expire and $T=2$ for the call to be exercised. After one period, the stock price can either be $u S_{(0)}$ or $d S_{(0)}$ between the first and second periods.

The stock price can once again go up by $u$ or down by $d$, so the possible prices of the stock for the two periods are $u u S_{(0)}$ or $u^{2} S_{(0)}, u d S_{(0)} d^{2} S_{(0)}$ or $d d S_{(0)}$.

We can also trace the movement of the stock price from $T=1$ to $T=2$ from the perspective of tossing a coin, and the outcome of the coin toss determines the price of the stock at $T=1$.

We assume the coin toss need not to be fair which implies the probability of head need not to be half .We assume only that the probability of head, which we take to be $\frac{\hat{A}}{\hat{A}+\hat{B}}>0$ and the probability of getting a tail take to be $1-\frac{\hat{A}}{\hat{A}+\hat{B}}>0$. Now if we repeatedly toss the coin and whenever we get the head the stock price moves up by the factor $u$ whereas whenever we get a tail, the stock price moves down by a factor $d$.

When $S_{(0)}$ is the initial value of the stock, $u S_{(0)}$ is the possible stock value at time $T=1$, when the price goes up, $u^{2} S_{(0)}$ denote the value of the stock at time $T=2$. When the stock price gets to the peak, $d S_{(0)}$ is the value of the stock when the price goes down at time $T=1$ and $d^{2} S_{(0)}$ is the value of the stock when the price goes down to the lowest price at time $T=2$.

Where $C_{(0)}$ is the unknown call price.

Hence $C_{u u}=C_{2}(H H), \quad C_{u d}=C_{2}(T H)$ and $C_{d d}=$ $C_{2}(T T)$ are payoff call at expiration $T=2$.

Hence $C_{u}$ and $C_{d}$ can be determined by defining $C_{u}=C_{1}(H)$ and $C_{d}=C_{1}(T)$, with one period to expiration when the stock price is either $u S_{(0)}=S_{1}(H)$ or $d S_{(0)}=S_{1}(T)$.

Cox et al [3] and Chandra et al [6] gave expression for $C_{u}$ and $C_{d}$ respectively as

$$
\begin{aligned}
C_{u} & =\frac{1}{R}\left[\hat{p} C_{u u}+(1-\hat{p}) C_{u d}\right] . \\
C_{d} & =\frac{1}{R}\left[\hat{p} C_{u d}+(1-\hat{p}) C_{d d}\right] .
\end{aligned}
$$

Herein, we try to make use of Wealth equation to derive the expression for $C_{u}$ and $C_{d}$. Thus

By lemma 2.3 Wealth equation gives

$$
V_{t+1}=a_{t} S_{t}+1+r\left[C_{t}-a_{t} S_{t}\right]=C_{t+1},
$$

for $t=T-1, T-2, \ldots$

Then

$$
C_{(t+1)}=V_{t+1}
$$

Thus

$$
C_{t+1}=C_{2}=\left\{\begin{array}{l}
C_{u u}=C_{2}(H H) \\
C_{u d}=C_{2}(H T) \\
C_{d u}=C_{2}(T H) \\
C_{d d}=C_{2}(T T)
\end{array},\right.
$$

which implies that

$$
\begin{gathered}
V_{2}=\left\{\begin{array}{l}
a_{1} S_{2}(H H)+1+r\left[C_{1}(H)-a_{1} S_{1}(H)\right]=C_{2}(H H) \\
a_{1} S_{2}(H T)+1+r\left[C_{1}(H)-a_{1} S_{1}(H)\right]=C_{2}(H T) \\
a_{t} S_{2}(T H)+1+r\left[C_{1}(T)-a_{1} S_{1}(T)\right]=C_{2}(T H) \\
a_{1} S_{2}(T T)+1+r\left[C_{1}(T)-a_{1} S_{1}(T)\right]=C_{2}(T T)
\end{array}\right. \\
a_{1}=\frac{C_{2}(H H)-C_{2}(H T)}{S_{2}(H H)-S_{2}(H T)}=\frac{C_{u u}-C_{u d}}{U S_{1}(H)-d S_{1}(H)} \\
=\frac{C_{u u}-C_{u d}}{(U-d) S_{1}(H)},
\end{gathered}
$$

Where $a_{1}$ is the hedging formula.

Substitute (27)in (23) gives

$$
\begin{aligned}
& a_{1} u S_{1}(H)+1+r C_{u}-1+r S_{1}(H)=C_{u u} \\
& \Rightarrow 1+r C_{u}+\frac{C_{u u}-C_{u d} S_{1}(H)[u-1+r]}{(u-d) S_{1}(H)}=C_{u u} \\
& \Rightarrow 1+r C_{u}+\frac{C_{u u}[u-1+r]}{(u-d)}-\frac{C_{u d}[u-1+r]}{(u-d)}=C_{u u} \\
& \Rightarrow 1+r C_{u} \frac{\hat{B}}{\hat{A}+\hat{B}} C_{u u}-\frac{\hat{B}}{\hat{A}+\hat{B}} C_{u d}=C_{u u} \\
& \Rightarrow 1+r C_{u}=C_{u u}-\frac{\hat{B}}{\hat{A}+\hat{B}} C_{u u}+\frac{\hat{B}}{\hat{A}+\hat{B}} C_{u d} \\
& =\left(1-\frac{\hat{A}}{\hat{A}+\hat{B}}\right) C_{u u}+\frac{\hat{A}}{\hat{A}+\hat{B}} C_{u d} \\
& C_{u}=\frac{1}{1+r}\left[\frac{\hat{A}}{\hat{A}+\hat{B}} C_{u u}+\frac{\hat{B}}{\hat{A}+\hat{B}} C_{u d}\right] .
\end{aligned}
$$

Now solving also equation (25) and (26) we obtain the value of $a_{1}$ and $C_{1}(T)=C_{d}$ as

$$
a_{1}=\frac{C_{2}(H T)-C_{2}(T T)}{S_{2}(T H)-S_{2}(T T)}=\frac{C_{u d}-C_{d d}}{U S_{1}(T)-d S_{1}(T)}
$$

and Substituting $a_{1}$ into (25) we have

$$
C_{d}=\frac{1}{1+r}\left[\frac{\hat{A}}{\hat{A}+\hat{B}} C_{u d}+\frac{\hat{B}}{\hat{A}+\hat{B}} C_{d d}\right] .
$$

Where

$$
\frac{\hat{A}}{\hat{A}+\hat{B}}=\frac{1+r-d}{u-d} \text { and } \frac{\hat{B}}{\hat{A}+\hat{B}}=\frac{u-1-r}{u-d},
$$

which are called risk neutral probabilities.

Substituting equation (28) and (30) into equation (18) we have 


$$
C_{(0)}=\frac{1}{1+r}\left[\begin{array}{l}
\frac{\hat{A}}{\hat{A}+\hat{B}}\left(\frac{1}{1+r}\right) \frac{\hat{A}}{\hat{A}+\hat{B}} C_{u u}+\frac{\hat{B}}{\hat{A}+\hat{B}} C_{u d} \\
+\frac{\hat{B}}{\hat{A}+\hat{B}}\left(\frac{1}{1+r}\right) \frac{\hat{A}}{\hat{A}+\hat{B}} C_{u d}+\frac{\hat{B}}{\hat{A}+\hat{B}} C_{d d}
\end{array}\right]
$$

or

$$
\begin{aligned}
& C_{(0)}=\frac{1}{1+r}\left[\begin{array}{l}
\frac{1}{1+r}\left(\frac{\hat{A}}{\hat{A}+\hat{B}}\right)^{2} C_{u u} \\
+2 \frac{\hat{A}}{\hat{A}+\hat{B}} \frac{\hat{B}}{\hat{A}+\hat{B}} C_{u d}+\left(\frac{\hat{B}}{\hat{A}+\hat{B}}\right)^{2} C_{d d}
\end{array}\right] \\
& =\frac{1}{1+r^{2}}\left[\begin{array}{l}
\left(\frac{\hat{A}}{\hat{A}+\hat{B}}\right)^{2} C_{u u}+2 \frac{\hat{A}}{\hat{A}+\hat{B}} \frac{\hat{B}}{\hat{A}+\hat{B}} C_{u d} \\
+\left(\frac{\hat{B}}{\hat{A}+\hat{B}}\right)^{2} C_{d d}
\end{array}\right]
\end{aligned}
$$

where $C_{u u} C_{u d}$ and $C_{d d}$ are the payoff of the stock at $t=2$.

Using the Dwass identity to generalized the payoff values of the stock as follows

$$
C_{N}(x)=\operatorname{Max}\left[U^{x} d^{N-x} S_{(0)}-N, 0\right]
$$

where $x=0,1,2 \ldots, N$ and $x$ depends on the factor $u$.

Now

$$
\begin{aligned}
& C_{(0)}=\frac{1}{1+r^{2}}\left[\begin{array}{l}
\left(\frac{\hat{A}}{\hat{A}+\hat{B}}\right)^{2} C_{u u}+2 \frac{\hat{A}}{\hat{A}+\hat{B}} 1-\frac{\hat{A}}{\hat{A}+\hat{B}} C_{u d} \\
+\left(1-\frac{\hat{A}}{\hat{A}+\hat{B}}\right)^{2} C_{d d}
\end{array}\right] \\
& \times \frac{1}{(1+r)^{2}}\left[\begin{array}{l}
\left(\frac{\hat{A}}{\hat{A}+\hat{B}}\right)^{2} C_{2}(2)+2 \frac{\hat{A}}{\hat{A}+\hat{B}} 1 \\
-\frac{\hat{A}}{\hat{A}+\hat{B}} C_{2}(1)+\left(\frac{\hat{B}}{\hat{A}+\hat{B}}\right)^{2} C_{2}(0)
\end{array}\right] \\
& =\frac{1}{(1+r)^{2}}\left[\begin{array}{l}
\left(\frac{\hat{A}}{\hat{A}+\hat{B}}\right)^{2}+2 \frac{\hat{A}}{\hat{A}+\hat{B}} 1 \\
-\frac{\hat{A}}{\hat{A}+\hat{B}}+\left(1-\frac{\hat{A}}{\hat{A}+\hat{B}}\right)^{2}
\end{array}\right] \\
& \times \operatorname{Max}\left[U^{X} d^{N-x} S_{(0)}-K, 0\right]
\end{aligned}
$$

Since $\mathrm{R}=1+r$ we have

$$
\begin{aligned}
C_{(0)}= & \frac{1}{R^{2}}\left[\left(\frac{\hat{A}}{\hat{A}+\hat{B}}\right)^{2}+2 \frac{\hat{A}}{\hat{A}+\hat{B}} 1-\frac{\hat{A}}{\hat{A}+\hat{B}}+\left(\frac{\hat{B}}{\hat{A}+\hat{B}}\right)^{2}\right] \\
& \times \operatorname{Max}\left[u^{x} d^{N-x} S_{(0)}-K, 0\right] \\
= & \frac{1}{R^{2}}\left(\frac{\hat{A}}{\hat{A}+\hat{B}}+\frac{\hat{B}}{\hat{A}+\hat{B}}\right)^{2} \operatorname{Max}\left[u^{x} d^{N-x} S_{(0)}-K, 0\right] .
\end{aligned}
$$

By Binomial theory expansion, we have that

$$
\begin{aligned}
& \left(\frac{\hat{A}}{\hat{A}+\hat{B}}+1-\frac{\hat{A}}{\hat{A}+\hat{B}}\right)^{N} \\
& =N_{C 0}\left(\frac{\hat{A}}{\hat{A}+\hat{B}}\right)^{N}\left(\frac{\hat{B}}{\hat{A}+\hat{B}}\right)^{0}+N_{C 1}\left(\frac{\hat{A}}{\hat{A}+\hat{B}}\right)^{N-1}\left(\frac{\hat{B}}{\hat{A}+\hat{B}}\right)^{1} \\
& +N_{C 2}\left(\frac{\hat{A}}{\hat{A}+\hat{B}}\right)^{N-2}\left(\frac{\hat{B}}{\hat{A}+\hat{B}}\right)^{2}+\ldots \ldots \ldots N_{C N} \\
& +\left(\frac{\hat{A}}{\hat{A}+\hat{B}}\right)^{N-N}\left(\frac{\hat{B}}{\hat{A}+\hat{B}}\right)^{N} .
\end{aligned}
$$

Better still

$$
\left(\frac{\hat{A}}{\hat{A}+\hat{B}}+1-\frac{\hat{A}}{\hat{A}+\hat{B}}\right)^{N}=\sum_{x=0}^{N}\left(\begin{array}{l}
N \\
x
\end{array}\right) \frac{\hat{A}^{x} \hat{B}^{N-x}}{(\hat{A}+\hat{B})^{(x)}(\hat{A}+\hat{B})^{(N-x)}} .
$$

Therefore

$$
\begin{aligned}
& C_{(0)}=\frac{1}{R^{N}} \sum_{x=0}^{n}\left(\begin{array}{l}
n \\
x
\end{array}\right) \frac{\hat{A}^{x} \hat{B}^{N-x}}{(\hat{A}+\hat{B})^{(x)}(\hat{A}+\hat{B})^{(N-x)}} \\
& =\frac{1}{R^{N}} \sum_{x=0}^{n}\left(\begin{array}{l}
N \\
x
\end{array}\right) \frac{\hat{A}^{x} \hat{B}^{N-x}}{(\hat{A}+\hat{B})^{(N)}} \operatorname{Max}\left[U^{x} d^{N-x} S_{(0)}-K, 0\right] .
\end{aligned}
$$

Which implies

$$
C_{(0)}=\frac{1}{R^{N}} \sum_{x=0}^{N}\left(\begin{array}{c}
N \\
x
\end{array}\right) \frac{\hat{A}^{X} \hat{B}^{N-x}}{(\hat{A}+\hat{B})^{(N)}} C_{N}(x) .
$$

Remark 3.1: $\frac{\hat{A}}{\hat{A}+\hat{B}}$ and $1-\frac{\hat{A}}{\hat{A}+\hat{B}}$ used in this paper is in extension with $\hat{p}$ and $\hat{q}$ used in Cox et al [4] and Chandral et al [2], and satisfies the property of a probability which implies that $\frac{A}{A+B}+1-\frac{A}{A+B}=1$.

$$
0<\frac{\hat{A}}{\hat{A}+\hat{B}}<\frac{\hat{B}}{\hat{A}+\hat{B}}<1 A+B \text { gives the chance of the up }
$$
and down movement of the stock and call prices.

The European put option follows exactly the same derivation as the European call option, by induction method we obtain

$$
P_{(0)}=\frac{1}{R^{N}} \sum_{x=0}^{N}\left(\begin{array}{l}
N \\
x
\end{array}\right) P_{N}(x)
$$

with the following pay-off values

$$
\begin{aligned}
& P_{u u}=P_{2}(2)=\operatorname{Max}\left[K-U^{x} d^{N-x} S(0), 0\right], \\
& P_{u d}=P_{2}(1)=\operatorname{Max}\left[K-U^{X} d^{N-x} S(0), 0\right], \\
& P_{d d}=P_{2}(0)=\operatorname{Max}\left[K-U^{X} d^{N-x} S(0), 0\right],
\end{aligned}
$$


Generally $P_{N}(x)=\operatorname{Max}\left[K-U^{x} d^{N-x} S(0), 0\right]$ for all $\mathrm{T}=2$ and $x=0,1,2 \ldots N$.

\section{Numerical Illustration}

The following illustrative examples are used to validate the theoretical results

Example 4.1: Let $S_{(0)}=100, K=100, u=1.2, d=$ $0.8, r=10 \%$. Also set $\mathrm{R}=1+r$, then

$$
\begin{aligned}
& \frac{\hat{A}}{\hat{A}+\hat{B}}=\frac{R-d}{u-d} \frac{1.1-0.8}{1.2-0.8}=\frac{75}{100} \\
& \text { and } \frac{\hat{B}}{\hat{A}+\hat{B}}=1-\frac{\hat{A}}{\hat{A}+\hat{B}}=1-\frac{75}{100}=\frac{25}{100} .
\end{aligned}
$$

Given also the payoff to be of the form

$$
C_{N}(x)=\operatorname{Max}\left[U^{x} d^{N-x} S(0)-K, 0\right]
$$

where $x=0,1,2 . . N$, then $C_{2}(2)=C_{u u}=44, C_{2}(1)=$ $C_{u d}=0$, and $C_{2}(0)=C_{d d}=0$.

Using the above information, we obtain

$$
\begin{aligned}
& C_{u}=\frac{1}{R}\left[\frac{\hat{A}}{\hat{A}+\hat{B}} C_{u u}+\frac{\hat{B}}{\hat{A}+\hat{B}} C_{u d}\right]=\$ 30 \\
& C_{d}=\frac{1}{R}\left[\frac{\hat{A}}{\hat{A}+\hat{B}} C_{u d}+\frac{\hat{B}}{\hat{A}+\hat{B}} C_{d d}\right]=\$ 0 .
\end{aligned}
$$

$C_{u}$ and $C_{d}$ values are the possible prices of the option before the expiration .

Using the same information to find the price of European call and put option as follows

$$
S_{(0)}=100, K=100, u=1.2, d=0.8, r=10 \%
$$

per year and time to expiry $T=2$.

Using the model for call option which is as given as

$$
C_{(0)}=\frac{1}{R^{N}} \sum_{x=0}^{n}\left(\begin{array}{l}
N \\
x
\end{array}\right) \frac{\hat{A}^{x} \hat{B}^{N-x}}{(\hat{A}+\hat{B})^{(N)}} C_{N}(x)
$$

with a little simplification we obtain

$$
C_{(0)}=\frac{1}{R^{N}} \sum_{x=0}^{n}\left(\begin{array}{c}
N \\
x
\end{array}\right) \frac{\hat{A}^{x} \hat{B}^{n-x}}{(\hat{A}+\hat{B})^{x}(\hat{A}+\hat{B})^{n-x}} C_{N}(x) .
$$

Let $R=r+1=10 \%+1=\frac{10}{100}=0.1+1=1.1$

$$
\frac{A}{A+B}=\frac{R-d}{u-d}=\frac{75}{100} \text { and } 1-\frac{A}{A+B}=\frac{B}{A+B}=\frac{25}{100} \text {. }
$$

Then the possible ending values for the call option after $T=2 ; C_{t}(x)=\operatorname{Max}\left[U^{x} d^{N-x} S(0)-k, 0\right]$ where $x=$ $0,1,2 . . N$ are $C_{2}(2)=C_{u u}=44, \quad C_{t}(1)=C_{u d}=0$, $C_{2}(0)=C_{d d}=0$ and $C_{(0)}=\$ 20.45$

Now for the price of a European put option using the same data $S_{(0)}=100, K=100, u=1.2, d=0.8, r=$ $10 \%$ per year and time to expiry $\mathrm{T}=2$. Let $\mathrm{R}=r+1=1.1$. Then

$$
\frac{A}{A+B}=\frac{R-d}{u-d}=\frac{75}{100} \text { and } 1-\frac{A}{A+B}=\frac{B}{A+B}=\frac{25}{100} .
$$

with following pay-off values $P_{u u}=P_{t}(2)=0, P_{u d}=$ $P_{t}(1)=4$ and $P_{d d}=P_{t}(0)=36$. Given the model of the form

$$
P_{(0)}=\sum_{x=0}^{n}\left(\begin{array}{c}
N \\
x
\end{array}\right)\left(\frac{A}{A+B}\right)^{x}\left(\frac{B}{A+B}\right)^{N-x} P_{(t)}(x),
$$

then $P_{(0)}=\$ 3.10$.

Example 4.2: Now assume that $S_{(0)}=100, K=100$, $r=7 \%, T=3, u=1.1$ and $d=0.9$ in addition to example 4.1 above.

$$
C_{(0)}=\frac{1}{R^{N}} \sum_{x=0}^{n}\left(\begin{array}{l}
N \\
x
\end{array}\right) \frac{\hat{A}^{x} \hat{B}^{n-x}}{(\hat{A}+\hat{B})^{X}(\hat{A}+\hat{B})^{n-x}} C_{N}(x) .
$$

Then the possible ending values for the call option after $T=3$ are $C_{3}(3)=33.10, C_{3}(2)=C_{u d}=8.90, C_{3}(1)=$ $C_{d d}=0$ and $C_{3}(0)=C_{d d}=0$.

By (32) $C_{(0)}=\$ 18.96$.

Now for the price of a European put option using the same data we have; $S_{(0)}=100, K=100, r=7 \%, T=$ 3, $u=1.1$ and $d=0, \mathrm{R}=1.07, \frac{A}{A+B}=\frac{75}{100}$ and $1-\frac{A}{A+B}=\frac{25}{100}$. Then following pay-off values are obtained $P_{3}(3)=0, \quad P_{3}(2)=0, \quad P_{3}(1)=10.9$, and $P_{3}(0)=27.10$. By (33), $P_{(0)}=\$ 0.68$.

Example 4.3: Given that $S_{(0)}=80, K=100, u=1.2, d=$

\begin{tabular}{|c|c|c|c|c|c|c|c|c|}
\hline \multicolumn{9}{|c|}{$\boldsymbol{S}_{(\mathbf{0}) \boldsymbol{i}}=$ Stocks value, $\boldsymbol{K}_{\boldsymbol{i}}=$ stricks price, $\boldsymbol{T}_{\boldsymbol{i}}=$ Expiration dates, $\boldsymbol{R}_{\boldsymbol{i}}=\boldsymbol{r}+\mathbf{1}, \boldsymbol{r}=$ interest rate, $\boldsymbol{C}_{\boldsymbol{i}(\mathbf{0})}=$ Calls Price } \\
\hline$i$ & $T_{i}$ & $S_{(0) i}$ & $K_{i}$ & $u_{i}$ & $d_{i}$ & $R_{i}=r+1$ & $\boldsymbol{C}_{\boldsymbol{i}(\mathbf{0})}$ & $\boldsymbol{P}_{\boldsymbol{i}(0)}$ \\
\hline \multirow{2}{*}{1} & \multirow{2}{*}{1} & $\$ 100$ & $\$ 80$ & 1.2 & 0.8 & 1.1 & $\$ 27.27$ & $\$ 0$ \\
\hline & & $\$ 80$ & $\$ 100$ & 1.2 & 0.8 & 1.1 & $\$ 0$ & $\$ 10.91$ \\
\hline 2 & 2 & $\$ 100$ & $\$ 100$ & 1.2 & 0.8 & 1.1 & $\$ 20.45$ & $\$ 3.10$ \\
\hline \multirow{2}{*}{3} & \multirow{2}{*}{3} & $\$ 80$ & $\$ 100$ & 1.2 & 0.8 & 1.1 & $\$ 12.13$ & $\$ 7.28$ \\
\hline & & $\$ 100$ & $\$ 80$ & 1.2 & 0.8 & 1.1 & $\$ 40.42$ & $\$ 0.76$ \\
\hline
\end{tabular}
$0.8, r=10 \%$ and $T=3$, we obtain $R=1.1$, $\frac{A}{A+B}=0.75$ and $\frac{B}{A+B}=0.25$. Then the possible ending values for the call option after $T=3$ are given as; $C_{3}(3)=38.40, C_{3}(2)=0, C_{3}(1)=C_{d d}=0$, and $C_{3}(0)=$ $C_{d d}=0$. By (32) $C_{(0)}=\$ 12.13$. For the price of a European put option using the same data, we obtain the following; $S_{(0)}=80, K=100, u=1.2, d=0.8, r=$ $10 \%$ and $T=3$, with following pay-off values $P_{3}(3)=0$, $P_{3}(2)=7.84, P_{3}(1)=38.56$, and $P_{3}(0)=59.20$. And by (33), $P_{(0)}=\$ 7.28$.

Table 1. Calls and Puts price Paper 
Table 1 above shows how well the generalized Binomial distribution associate with finance terms, can be used to evaluate call and put options, using equation (32) and (33) for range of values of $T_{i}, \mathrm{~S}_{\mathrm{i}}$ and $K_{i}$

\section{Monitoring the Behaviour of Call and Put Option}

Table 2 below summarizes the variable and their predicted effect on call and put prices from the application point of view, by keeping other variables constant.

Case 1: Varying interest rates: Keeping the following variables constant $u=1.2, d=0.8, t=2, K=100$ and $S_{(0)}=100$.

Table 2. DECREASE IN INTEREST RATE

\begin{tabular}{|c|c|c|c|c|c|}
\hline$i$ & $r \%$ & $\frac{A}{A+B} \%$ & $\frac{B}{A+B} \%$ & $C_{i(0)}$ & $P_{i(0)}$ \\
\hline 1 & $10 \%$ & $75 \%$ & $25 \%$ & $\$ 20.45$ & $\$ 3.10$ \\
\hline 2 & $9 \%$ & $73 \%$ & $27 \%$ & $\$ 19.70$ & $\$ 3.53$ \\
\hline 3 & $8 \%$ & $70 \%$ & $30 \%$ & $\$ 18.43$ & $\$ 4.21$ \\
\hline 4 & $7 \%$ & $68 \%$ & $32 \%$ & $\$ 17.85$ & $\$ 4.76$ \\
\hline 5 & $6 \%$ & $65 \%$ & $35 \%$ & $\$ 16.60$ & $\$ 5.46$ \\
\hline 6 & $5 \%$ & $63 \%$ & $37 \%$ & $\$ 15.88$ & $\$ 5.96$ \\
\hline 7 & $4 \%$ & $60 \%$ & $40 \%$ & $\$ 14.67$ & $\$ 7.11$ \\
\hline
\end{tabular}

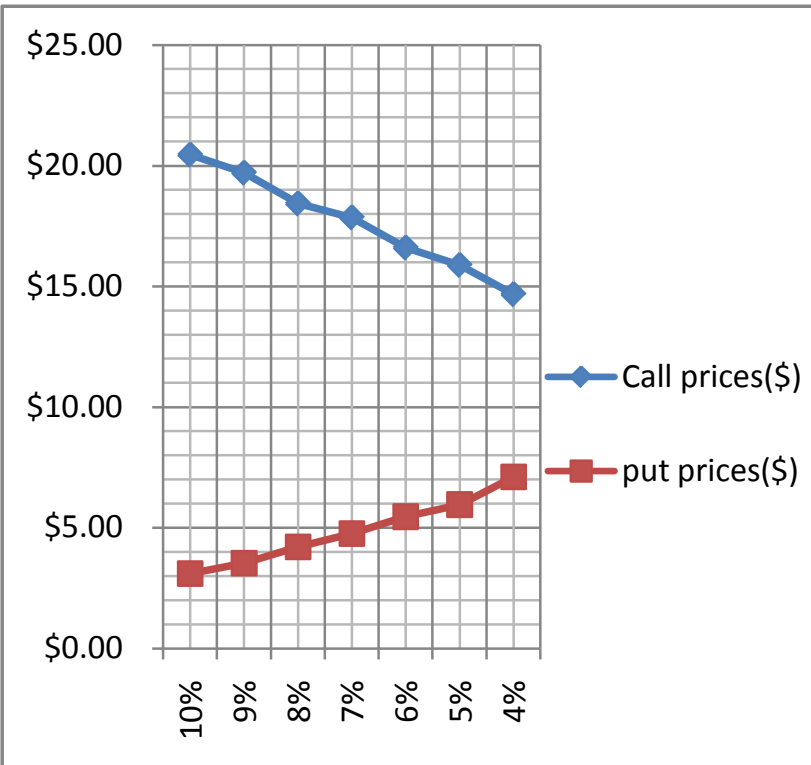

Figure 1. A graph of calls,puts price against interest rate

Figure 1 and Table 2 above show that decrease in interest rate leads to decrease in calls price, and increase in puts price.

Case 2: Varying the Strick price: Keeping the following variables constant, $u=1.2, d=0.8, T=2, S(0)=$ $100, R=r+1=10 \%+1$.

Table 3. INCREASE IN STRIKE PRICES

\begin{tabular}{|c|c|c|c|c|c|}
\hline$i$ & $K_{i}$ & $\frac{A}{A+B}$ & $\frac{B}{A+B}$ & $C_{i(0)}$ & $P_{i(0)}$ \\
\hline 1 & 100 & $75 \%$ & $25 \%$ & $\$ 20.45$ & $\$ 3.10$ \\
\hline 2 & 105 & $75 \%$ & $25 \%$ & $\$ 18.13$ & $\$ 4.90$ \\
\hline 3 & 110 & $75 \%$ & $25 \%$ & $\$ 15.18$ & $\$ 6.71$ \\
\hline 4 & 115 & $75 \%$ & $25 \%$ & $\$ 13.48$ & $\$ 8.52$ \\
\hline
\end{tabular}

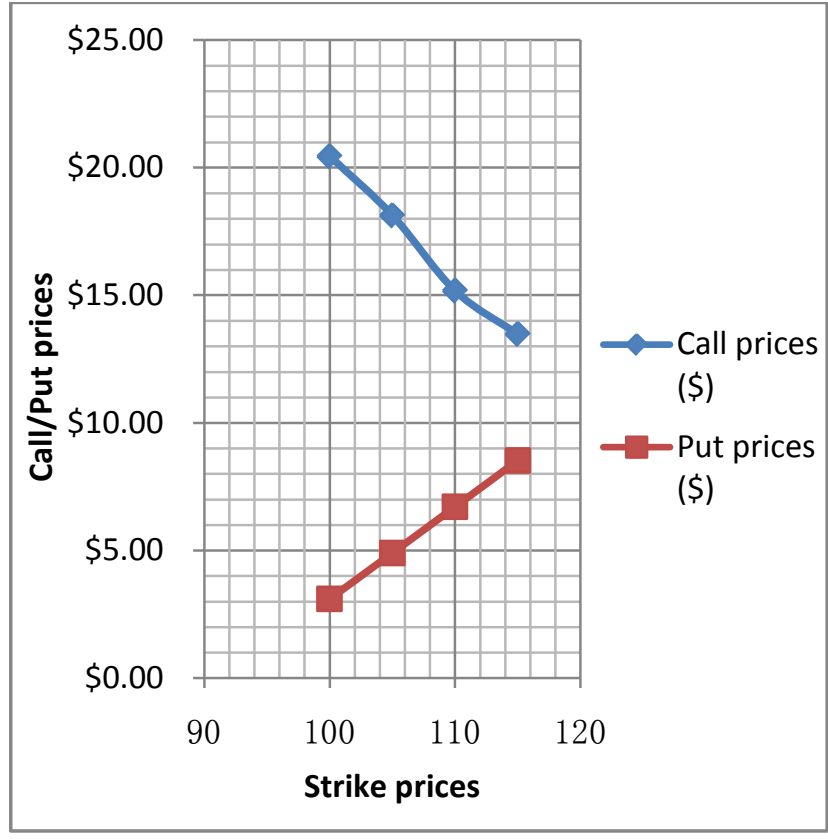

Figure 2. A graph of calls, puts price against strike prices

Figure 2 and Table 3 Show that increase in strike prices will leads to decrease in calls price and increase in puts price.

Case 3: Varying the Expiration date: Keeping also the following variables constant $u=1.2, d=0.8, K=100$, $S(0)=100, R=r+1=10 \%+1$.

Table 4. DECREASE IN EXPIRATION DATE

\begin{tabular}{|c|c|c|c|c|c|}
\hline$i$ & $T_{i}$ & $\frac{A}{A+B}$ & $\frac{B}{A+B}$ & $C_{i(0)}$ & $P_{i(0)}$ \\
\hline 1 & 4 & $75 \%$ & $25 \%$ & $\$ 34.27$ & $\$ 4.01$ \\
\hline 2 & 3 & $75 \%$ & $25 \%$ & $\$ 26.09$ & $\$ 3.02$ \\
\hline 3 & 2 & $75 \%$ & $25 \%$ & $\$ 20.45$ & $\$ 2.28$ \\
\hline
\end{tabular}

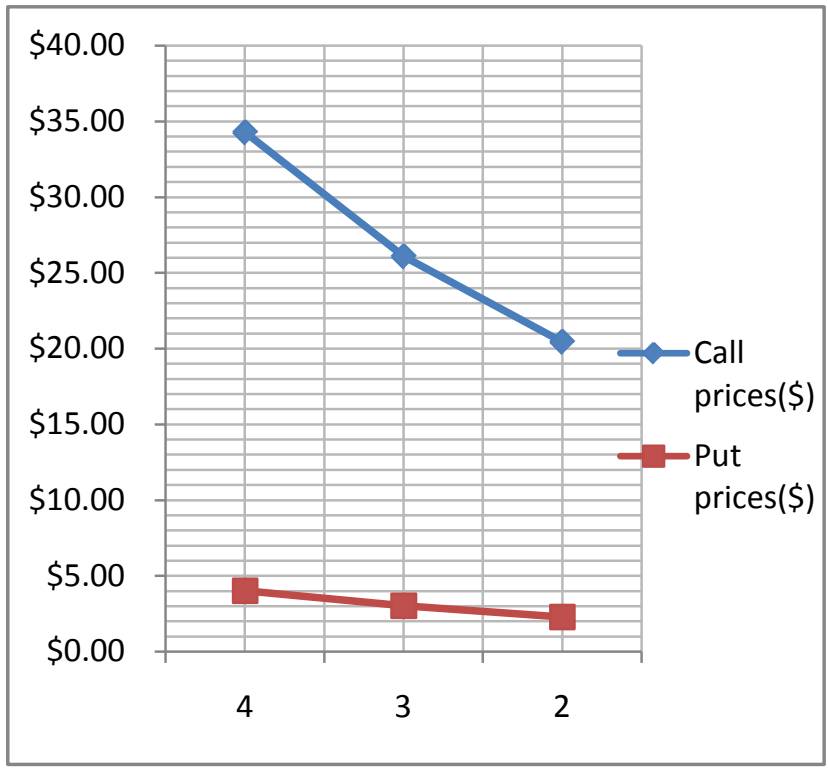

Figure 3. A graph of calls and puts price against Time.

Figure 3 and Table 4 show that decrease in expiration date leads to decrease in calls price and a slight decrease in puts price. 
Case 4: Varying the Stock price: Keeping also the following variables constant $u=1.2, d=0.8, K=100$, $R=r+1=10 \%+1$.

Table 5. INCREASE IN STOCK PRICES

\begin{tabular}{|c|c|c|c|c|c|}
\hline$i$ & $S_{i(0)}$ & $\frac{A}{A+B} \%$ & $\frac{B}{A+B} \%$ & $C_{i(0)}$ & $P_{i(0)}$ \\
\hline 1 & $\$ 80$ & $75 \%$ & $25 \%$ & $\$ 7.07$ & $\$ 9.71$ \\
\hline 2 & $\$ 85$ & $75 \%$ & $25 \%$ & $\$ 10.41$ & $\$ 8.05$ \\
\hline 3 & $\$ 90$ & $75 \%$ & $25 \%$ & $\$ 13.75$ & $\$ 7.75$ \\
\hline 4 & $\$ 95$ & $75 \%$ & $25 \%$ & $\$ 17.12$ & $\$ 5.75$ \\
\hline 5 & $\$ 100$ & $75 \%$ & $25 \%$ & $\$ 20.45$ & $\$ 3.10$ \\
\hline
\end{tabular}

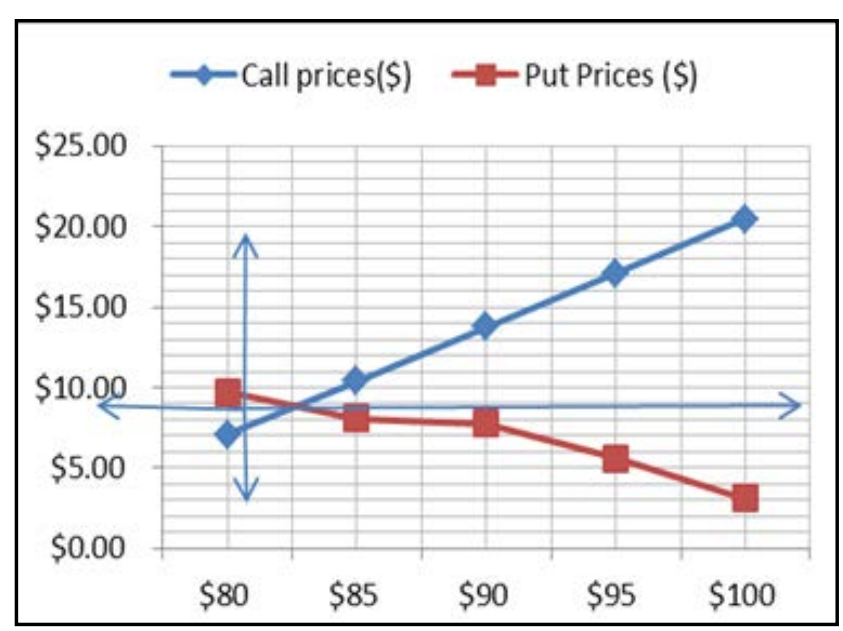

Figure 4. Graph of calls and puts price against the stock price.

Figure 4 and Table 5 show that increase in stock price leads to increase in calls price and decrease in puts price.

\section{Discussion of Results}

It is found that the problem of option price can be approached using generalized Binomial distribution associating it with finance terms which gives the same numerical results with Chandral et al [2] using the same information.

Table 1, It is clear that when the call option is in-themoney implies $\left(S_{(0) 1}>K_{1}\right)$ and $\left(S_{(0) 3}>K_{3}\right)$ the call option gets higher value, when the put option is in-themoney, implies $\left(S_{(0) 1}<K_{1}\right)$ and $\left(S_{(0) 3}<K_{3}\right)$ get higher value.When the call option is out-of -the money, implies $\left(S_{(0) 1}<K_{1}\right)$ and $\left(S_{3}<K_{3}\right)$ it loses value, put option is out -of -the money, implies $\left(S_{(0) 1}>K_{1}\right)$ and $\left(S_{(0) 3}>K_{3}\right)$ also loses value. This is in agreement with Adam [1] options that are in-the-money have a higher value compared to options that are out-of-the money.

Figure 1 and Table 2, show that increase in interest rate leads to increase in calls price and decrease in puts price .It is also observed that as the interest rate tends to zero, there will be a point of intersection of the prices, which will make both call and put of equal price. Which implies $C_{i(0)} \geq P_{i(0)}$. This agrees with Adam [1] when interest rate rise, a call option value will also rise and put option value will fall.
For Figure 2 and Table 3; when there is increase in stock price, call price decreases and put price will increase. It is also observed that as the strike price keeps increasing, there will be an equal price thereby having a point of intersection of prices. Chandral et al [2] have it that $C_{K i}(0)$ is a non increasing and $P_{k i}(0)$ is a non-decreasing function of $K$. Clearly from Table 3 and Figure $2 C_{i k(0)} \geq P_{i k(0)}$.

Figure 3 and Table 4 , shows that decrease in expiration will lead to decrease in both calls and puts price. This is in agreement with Nyustern [7] both calls and puts become more valuable as the time to expiration increase and loses more value as time decreases. It is observed in Figure 3 that the prices will always be in a parallel price form, meaning there will be no point of intersection of price. Implies $C_{i(0)}>P_{i(0)}$.

Figure 4 and Table 5 show that increase in stock prices leads to increase in calls price and decrease in puts price. From Figure 4, it is clear that when there is increase in stock prices there will be an equal price of call and put. (point of intersection), such that $C_{i S(0)}=P_{i S(0)}$. In general $C_{i S(0)} \geq P_{i S(0)}$. Nystern [7] an increase in the asset will increase the alue of the calls, puts on the other hand, becomes less valuable as the value of the asset increases. which agrees with the study.

\section{Conclusion}

Whenever stock price movement is confirmed to be discrete, in movement, the price of the option can be evaluated using generalized Binomial distribution (GBD). And the behaviour of the price of an option (call and put) is influence and dependent on the following.
i. The strike price $\mathrm{K}$
ii. The expire time $\mathrm{T}$
iii. The risk free rate $r$
iv. The underlying price $S_{(0)}$

\section{Acknowledgements}

Sincere thanks to Professor Francis Ogbonnaya Otunta. The erudite Vice Chancellor of Michael Okpara University of Agriculture, Umudike, Nigeria.

\section{References}

[1] B. Adam (2015). Factors that affect an option's price, (online) Available at http://the option prophet .com.

[2] S. Chandral, S. D. A. Mehra and R. Khemchandani (2013). An introduction to Financial Mathematics, pp 49-75, Narosa publishing house, New Delhi.

[3] F. I. Cheng and C. I. Alice (2010). Application of Binomial distribution to evaluate call option (finance).Springer link,pp1-10.

[4] J. C. Cox, S. A. Ross and M. Rubinson (1979). Option pricing journal of financial Economics pp1-11.

[5] L. Diderik. (2011). Financial theory,ECON4510.

[6] P. Jan, (2012). Stochastic calculus in finance Rostock, pp 25-296.

[7] A. D. Nyustern (2015). Binomial option pricing and model chapter5 pp1-5, www. stern.nyu.edu/adamodar/pdfiles/.option.

[8] R. Stockbridge (2008). The distcrete Binomial model for option pricing, program in Applied Mathematics, university of Arizona. 
[9] K. Teerapabolan (2012). A pointwise approximation of generalized Binomial Applied mathematics V0l6.

[10] N. Teddy (2012). The discrete time Binomial Asset pricing model. Available online.
[11] K. Teerapabolan, and P. Wongkasem (2008). Approximating a generalized Binomial by Binomial and poisson distribution, international journal of statistics and system. vol 3, pp113-124. 